\title{
Efectos de la Covid-19 en salud mental: rescatando la dignidad humana por medio del afecto*
}

\author{
Andrés Eduardo Aguirre Antúnez ${ }^{1,2,3}$
}

(D) http://orcid.org/0000-0001-5317-4459

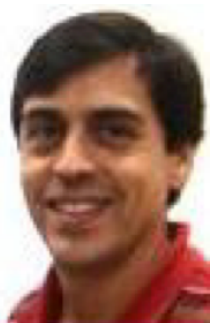

Antes de la pandemia Covid-19 nos encontrábamos en un ambiente universitario con constantes pedidos de ayuda por parte de estudiantes con trastornos psiquiátricos como ansiedad, depresión, trastornos bipolares, obsesivo-compulsivos, esquizofrenia, etc., y sufrimientos emocionales, como miedos, temores, angustias, soledad, desamparo, problemas existenciales, sensación de desarraigo y/o desesperación. En marzo del 2020 se suspendieron las actividades presenciales de la Universidad de São Paulo debido a la pandemia, pero las actividades remotas permitieron que la institución no se detuviera y siguiera sus actividades de enseñanza, investigación y atención psicológica.

La pandemia de Covid-19 genera un factor de estrés sin precedentes y la necesidad de apoyar a pacientes con enfermedades mentales graves, dado que es difícil mantener hábitos saludables como dieta y actividad física, autocontrol de condiciones crónicas de salud mental y física. El aumento de ansiedad y depresión puede generar riesgo de soledad y aislamiento en la población más vulnerable y en los profesionales de la salud, más aún si la pandemia es de larga duración ${ }^{(1)}$. Ante este escenario, investigadores recomiendan el trabajo multidisciplinario con alta inversión en investigación ${ }^{(2)}$ y una acción coordinada(3) que atienda las consecuencias sobre los grupos vulnerables.

Algunos efectos del Covid-19 que impactan la salud mental(4): temor a contaminarse y contaminar a otros, lo que desencadena reacciones agudas de estrés; necesidad de cuarentena, el cambio de rutina y confinamiento que culminan en sentimientos de desamparo, aburrimiento, ansiedad, angustia, irritabilidad y rabia por la pérdida de la libertad; duelo, depresión frente a las muertes sin los rituales de despedida que pueden aumentar el riesgo de suicidio; aquellos admitidos en UTI podrán desarrollar a futuro depresión grave, trastorno de estrés postraumático y otras condiciones psiquiátricas; las pérdidas económicas y de trabajo pueden transformar el estrés agudo en crónico y aumentar el riesgo de trastornos mentales. Cuando la angustia o la depresión es

\footnotetext{
* Apoyo financiero de la Fundação de Amparo à Pesquisa do Estado de São Paulo, São Paulo, SP, Brasil, Proceso no 18/19520-8 y del Conselho Nacional de Desenvolvimento Científico e Tecnológico (CNPq), Proceso no 302417/2018-4, Brasil.

1 Universidade de São Paulo, Instituto de Psicologia, Departamento de Psicologia Clínica, São Paulo, SP, Brasil.

2 Universidade de São Paulo, Pró-Reitoria de Graduação, Escritório de Saúde Mental, São Paulo, SP, Brasil.

${ }^{3}$ Universidade de São Paulo, Faculdade de Medicina, Instituto de Psiquiatria, São Paulo, SP, Brasil.
}

\section{Cómo citar este artículo}

Antúnez AEA. Effects of Covid-19 on mental health: Rescuing human dignity through affection. SMAD, Rev Eletrônica Saúde Mental Álcool Drog. 2021 abr.-jun.;17(2):4-6. doi: https://dx.doi.org/10.11606/issn.1806-6976.smad.2021.000166. 
incontrolable o impacta otros aspectos de la vida, como la función familiar o profesional, es necesario buscar ayuda profesional.

A partir del proyecto sobre suicidio entre estudiantes universitarios: estudio clínico y fenomenológico de orientación, prevención y terapéutica (FAPESP), hemos construido en la admisión y en las ruedas de charlas, presenciales y virtuales, en la Oficina de Salud Mental de la Prorrectoría de Graduación de la USP un conocimiento fruto de vivencias compartidas con la comunidad. En esta experiencia acogemos la complejidad humana: el dolor, la angustia, el miedo, la desesperación, el duelo, la esperanza o su ausencia, lo diferente, el cansancio, la falta de motivación. Ante una sociedad cuya comunicación virtual y tecnológica predomina, reflexionamos sobre la importancia de una educación básica que incluya la finitud (la muerte), la frustración y el duelo como procesos inherentes a la vida que es necesario dialogar y cuidar en interlocución con profesionales de la salud para que puedan madurar las personalidades así como también la dimensión espiritual, con la consolidación de la lucha contra la situación de crisis sanitaria.

Las perspectivas innovadoras, interdisciplinarias e internacionales son bienvenidas. La Filosofía nos ayuda a ampliar reflexiones, porque es una ciencia que trata el amor por la sabiduría, experimentado apenas por el ser humano consciente de su propia ignorancia(5). Disponemos de la cultura científica y de la humanística y en ellas, de dos importantes disciplinas, la psicología y la psicopatología, que se refieren a lo humano y a las patologías de lo psíquico sin que sea posible abandonarlas totalmente a las ciencias naturales siendo conveniente, de hecho, asumir una actitud crítica respecto de toda posición reduccionista del ser humano a uno de sus aspectos(6).

Es importante reconocer que, en la base de la cultura humanística, de las ciencias biológicas y exactas, se encuentra el ser humano y su dignidad. Ciencia significa saber y no se la puede reducir al paradigma de las ciencias naturales, físicas y orgánicas puesto que existen las ciencias humanas, que jamás podrán explicar el misterio del ser humano, aunque sí comprenderlo, respetarlo y tolerarlo. Así, podremos ayudar a otros en su integridad y complejidad, no a través de la exclusión, sino a partir del trabajo en integración y colaboración.

Vivimos un momento particular en la historia de la humanidad en que tendríamos que aunar esfuerzos y competencias para, de forma conjunta, ayudar a la humanidad y la sociedad, admitir los límites y el alcance de cada disciplina, acoger al diferente, al raro, al otro y reducir todo perjuicio y estigma personal y social.

Nosotros, seres humanos, no somos robots o máquinas pasivamente determinados. Somos afectados por los demás y por la vida que habita en nosotros. En afecto, nazco con el otro(7). Ser afectado no es una fragilidad o negatividad, sino más bien la posibilidad de una relación. En el afecto encontramos la fuerza y el dinamismo de la renovación de la cultura, en sus múltiples dimensiones: económica, política, estética, todas ellas profundamente afectadas por esta crisis universal.

Para combatir parte de las dificultades provenientes de la pandemia, es útil buscar a aquellos que sentimos, de modo intuitivo, que pueden ayudarnos, porque buscar ayuda es un gesto de sabiduría cuya acción amplía la comprensión, elaboración y la búsqueda de un nuevo sentido para la vida. La experiencia cotidiana al recibir estudiantes de la Universidad de São Paulo nos muestra que ellos quieren expresar sus sentimientos sin ser juzgados, sin prejuicios ni moralismos, de modo que desean encontrar relaciones que les devuelvan un sentir de pertenencia al mundo humano y así poder rescatar su dignidad y esperanza de afecto y reconocimiento mutuo.

Los jóvenes desean que se les reconozca, desean salir del aislamiento, la timidez y controlar sus ansiedades y tristezas. El cuidado pedagógico y personal es vital en su formación universitaria y humana, pero claman por cierta continuidad de cuidados y por el reconocimiento de su importancia personal y comunitaria. Es necesario creer en la potencia de la relación comprensiva, sensible e interesada por el otro, porque una relación significativa genera marcas en la vida del joven adulto y muestra que el principal antídoto para combatir los efectos psíquicos de la pandemia es la relación interpersonal equilibrada, solidaria y verdaderamente interesada por nuestro semejante, con confianza en su capacidad de resiliencia y afrontamiento.

\section{Referencias}

1 Druss BG. Addressing the COVID-19 Pandemic in Populations With Serious Mental Illness. JAMA Psychiatry. 2020;77(9):891-2. doi: http://dx.doi.org/10.1001/jamapsychiatry.2020.0894

2. Holmes EA, O'Connor RC, Perry VH, Tracey I, Wessely S, Arseneault L, et al. Multidisciplinary research priorities for the COVID-19 pandemic: a call for action for mental health science. Lancet Psychiatry. 2020;7(6):547-60. doi: http://dx.doi.org/10.1016/S2215-0366(20)30168-1

3. Leshner AL. Target student mental well-being. Science. 2021 Jan 22;371(6527):325. doi: http://dx.doi.org/10.1126/ science.abg5770 
4. Mari JJ, Oquendo MA. Mental health consequences of COVID-19: the next global pandemic. Trends Psychiatry Psychother. 2020 Sep [cited 2021 Feb 11];42(3):219-20. doi: https://doi.org/10.1590/2237-6089-2020-0081

5. Houaiss A. Dicionário Houaiss da Língua Portuguesa. Rio de Janeiro: Ed. Objetiva; 2001

6. Ales Bello A. O sentido do humano entre fenomenologia, psicologia e psicopatologia. São Paulo: Paulus; 2019.187 p

7. Martins F. Estátuas de anjos: para uma fenomenologia da vida e da clínica. Lisboa: Colibri; 2017.138 p

Copyright @ 2021 SMAD, Rev Eletrônica Saúde Mental Álcool Drog. Este es un artículo de acceso abierto distribuido bajo los términos de la Licencia Creative Commons CC BY.

Esta licencia permite a otros distribuir, mezclar, ajustar y construir a partir de su obra, incluso con fines comerciales, siempre que le sea reconocida la autoría de la creación original. Esta es la licencia más servicial de las ofrecidas. Recomendada para una máxima difusión y utilización de los materiales sujetos a la licencia. 